\section{A Fuzzy Decision Support System for Diagnosis of Some Liver Diseases in Educational Medical Institutions}

\author{
Ahmed Abd El-badie Abd Allah Kamel and Faten Abd El-Sattar Zahran \\ El-Mougi \\ Department of Computer Science, Faculty of Specific Education, Mansoura University, Egypt
}

\begin{abstract}
Decision support systems improve medical diagnosis and minimize diagnostic errors. Existing diagnostic systems are often complex and exhibit limited performance on liver diseases, particularly the liver cancer. This paper presents a fuzzy decision support system for helping students diagnose some human liver diseases in educational medical institutions. The proposed system aims to improve real medical diagnosis processes. The approach has three basic steps: 1) symptoms-based diagnosis, 2) liver function-based diagnosis, and 3) image processingbased diagnosis. The proposed system employs two artificial intelligence techniques: fuzzy logic and image processing. The first is used for diagnosing liver diseases based on the liver function tests, while the second is used for diagnosing liver diseases such as the liver cancer, hepatitis, liver cirrhosis, liver fibrosis, and fatty liver. The proposed system combines two methods: the Mamdani inference and simulation method used in the MATLAB17 fuzzy logic toolbox, and the gray level co-occurrence matrix, for extracting the features of the secondorder statistical texture of images acquired using computed tomography, magnetic resonance imaging, or ultrasound, for various liver diseases. Our results reveal a very good agreement between expert-made and system-made diagnoses, suggesting high accuracy.
\end{abstract}

Keywords: Decision support system, Fuzzy logic, Mamdani inference, Image processing, Liver diseases, Liver cancer

\section{Introduction}

Revised : Dec. 15, 2020

Accepted: Dec. 17, 2020

Correspondence to: Ahmed Abd El-badie Abd Allah Kamel

(ahmed_abdelbadie@mans.edu.eg)

(CThe Korean Institute of Intelligent Systems

(c) This is an Open Access article distributed under the terms of the Creative Commons Attribution Non-Commercial License (http://creativecommons.org/licenses/ by-nc/3.0// which permits unrestricted noncommercial use, distribution, and reproduction in any medium, provided the original work is properly cited.
Decision support systems (DSSs) are very useful for patients and medical experts in making decisions. It is a health information technology system designed for supplying physicians and other health professionals with decision support[1]. Clinical decision support systems (CDSSs) refer to programs that assist physicians in making decisions and show the capabilities of sophisticated reasoning for improving clinical decision making, thereby encouraging more effective care practices. Researchers designed early CDSSs on expert systems to program computers with rules that would allow ?thinking? as expert clinicians, compared with patients[2]. The liver is one of the most important organs for medication detoxification and reckless elimination from the demolition and reconstruction of red blood cells (RBCs) in the form of bile, the structure of blood-clotting parts, and sugar storage as glycogen. Moreover, the ordinance of sugar and fat metabolism are necessary functions of this organ. It also has the ability to perform fat ?digestion and exert defense with respect to foodstuff-related microbes 
and toxins[3]. The death numbers associated with liver diseases have been rising. Proper examination of liver diseases is needed for therapeutics and for mitigating their effects on health. DSSs can help experts diagnose diseases and can usefully supplement laboratory tests.

In addition, using DSSs decreases the disease diagnostics cost, decreases the decision error rate, and is time-efficient[4].

\section{Related Works}

Ahmad et al. suggested a novel multilayered Mamdani fuzzy inference system (Ml-MFIS) for diagnosing hepatitis B using a multi-layer Mamdani fuzzy inference system that classifies the different phases of hepatitis B, such as no hepatitis, severe hepatitis B virus (HBV), or chronic HBV. The reliability of the ML-MFIS expert system was 92.2\%[5]. Ahmad et al. proposed a hybrid intelligent approach that combines information acquisition methods and adaptive neuro-fuzzy inference systems (ANFIS), to diagnose fatal hepatitis disease. The performance of that approach was assessed using statistical methods, and the best results of the system validity, specificity, and sensitivity were $95.24 \%, 91.7 \%$, and $96.17 \%$, respectively. The results revealed that the developed intelligent system had a good diagnostic performance and could be used as a tool to diagnose hepatitis[6]. Mirmozaffari presented an expert system using an expert system shell for diagnosing the liver disease. In addition, block and Mockler charts were used, providing important information to the expert system[7]. Singh et al. suggested to use principal component analysis (PCA) and k-nearest neighbors (KNN) clustering for examining patients' data. Their model combined feature extraction and classification steps, performed by PCA and KNN, respectively. The results were compared using statistical metrics, including positive and negative predictive values [8]. Hashmi and Khan proposed a fuzzy control model for diagnosing a disease related to the human liver. The relative error between the simulated and calculated values was under $1.8 \%$, which was deemed acceptable[9]. Satarkar and Ali designed a fuzzy expert system to diagnose cirrhosis, which is the most common disease of the liver. This system was based on the sequential combination of the Bononcini score, including aspartate transaminase (AST), alanine transaminase (ALT) ratio, platelet count, and international normalized ratio (INR). The results showed that using fuzzy logic in designing the system enhanced the system's reasoning, even in the face of imprecise data. Incorporation of fuzzy logic into the expert system improved the system's performance[10]. Kim et al. introduced a method for determining the benefit signs from sensor arrays that had enormous sensing points, and the diagnosis of the liver cancer was done using machine learning algorithms, such as fuzzy neural networks and neural networks. Their results showed that the system could report the liver cancer with the accuracy of 99.19\% using 132 aptamers dependent on neural networks, and with the accuracy of $98.19 \%$ using 226 aptamers dependent on a fuzzy neural network[11]. Neshat et al. proposed a fuzzy Hopfield neural network for inferring the acuteness hepatitis. Their system used a fuzzy Hopfield neural network (HNN), and its performance was compared with that of a multilayer perceptron (MLP) neural network. Training was performed using a radial basis function (RBF) network, standard back propagation, the structure trained using the orthogonal least squares (OLS) algorithm, general regression neural networks (GRNNs), Bayesian networks with na?ve dependence and feature selection (BNNF), Bayesian networks with na?ve dependence (BNND), and HNNs. The results showed that the system had excellent performance, and the accuracy to diagnose the severity of hepatitis was $92.05 \%$ [12]. Adeli et al. introduced a hybrid method for diagnosing hepatitis diseases. The proposed method comprised two steps: 1) feature selection and 2) classification. Feature selection was performed using a genetic algorithm (GA) as an intelligent method for selecting features to reduce the overall number of used features. For classification, a basic intelligent classification method, ANFIS, was employed. The performance of the proposed method was compared with those of other methods from literature, in terms of the classification accuracy[13].

The above review suggests that most existing studies are mainly based on symptom diagnostics, while some studies used images for diagnosis. This makes our proposed system more distinctive and unique, as it allows to combining symptoms and images for diagnosis, and introduces diversity in the use of methodologies, algorithms, and tools for design.

\section{Proposed Approach}

The proposed system consists of three basic steps: 1) symptombased diagnosis, 2) liver function test-based diagnosis, and 3) image processing-based diagnosis. Figure 1 shows the framework of the proposed system.

\subsection{Symptom-Based Diagnosis}

Knowledge base in this study was based on the following stages:

Basic information about liver diseases and their associated symptoms was obtained using a database that contained records 


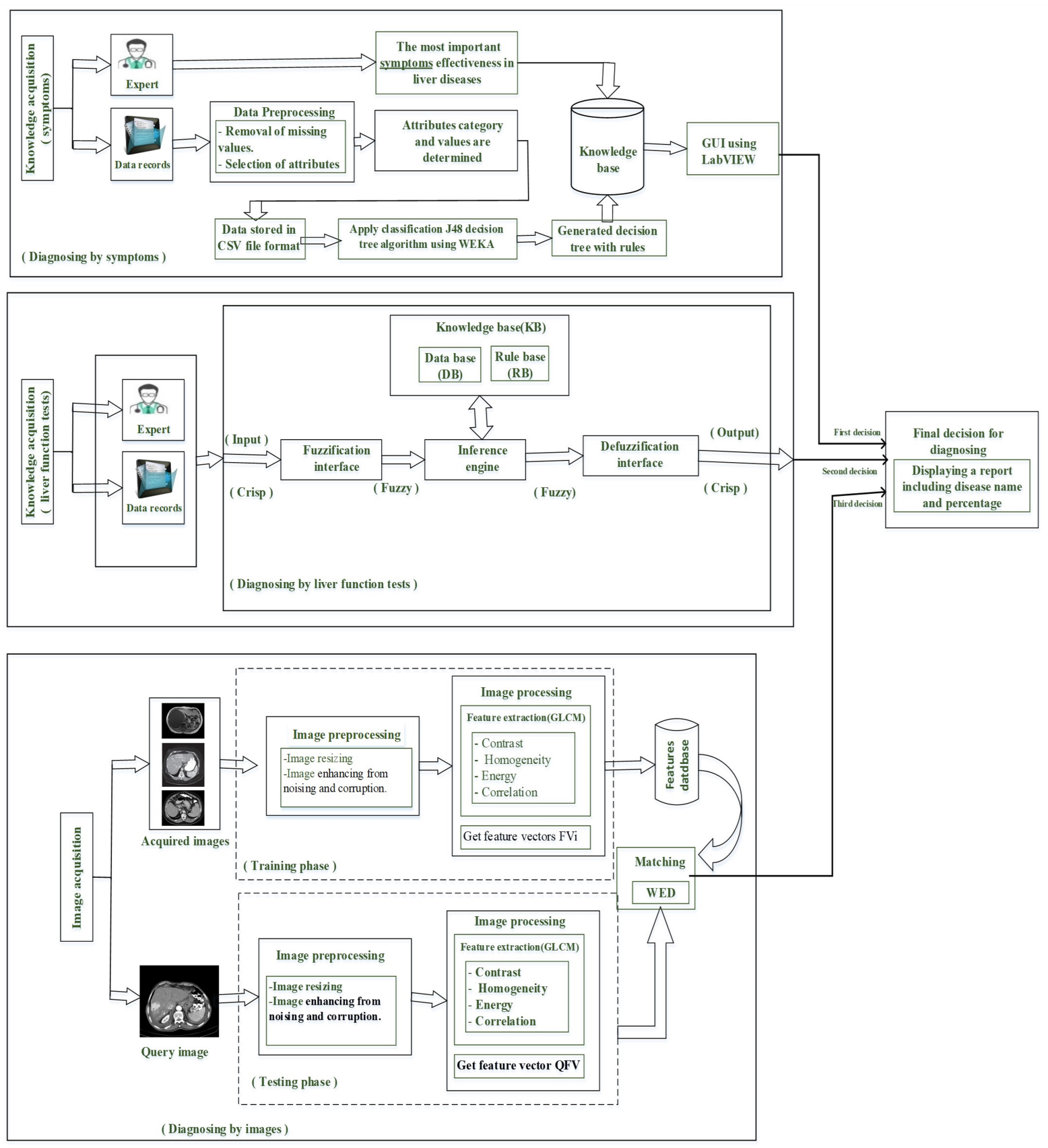

Figure 1. Schematic of the proposed system.

about patients with liver diseases that were previously diagnosed by more than one medical domain expert, at Mansoura University Hospital, Department of Internal Diseases. Figure 2 shows the flowchart for diagnosing the symptoms.

Through personal interviews with doctors and their opinions about the most important liver diseases, a new dataset called "diagnosing liver diseases" was created for the proposed system.

Data preprocessing was performed by removing missing values, ensuring that there are no incorrect data entered, and select- ing the attributes of the dataset that consisted of 100 instances derived from the database of the hospital including fourteen symptoms, listed in Table 1.

The database file was converted to a comma-separated value (CSV) file, and was used as a ?training dataset, where the datamining process was performed in the Waikato environment for ?knowledge analysis (WEKA 3.7), an open-source Javabased platform. This platform contains many machine learning algorithms for mining data, including clustering, classification, 


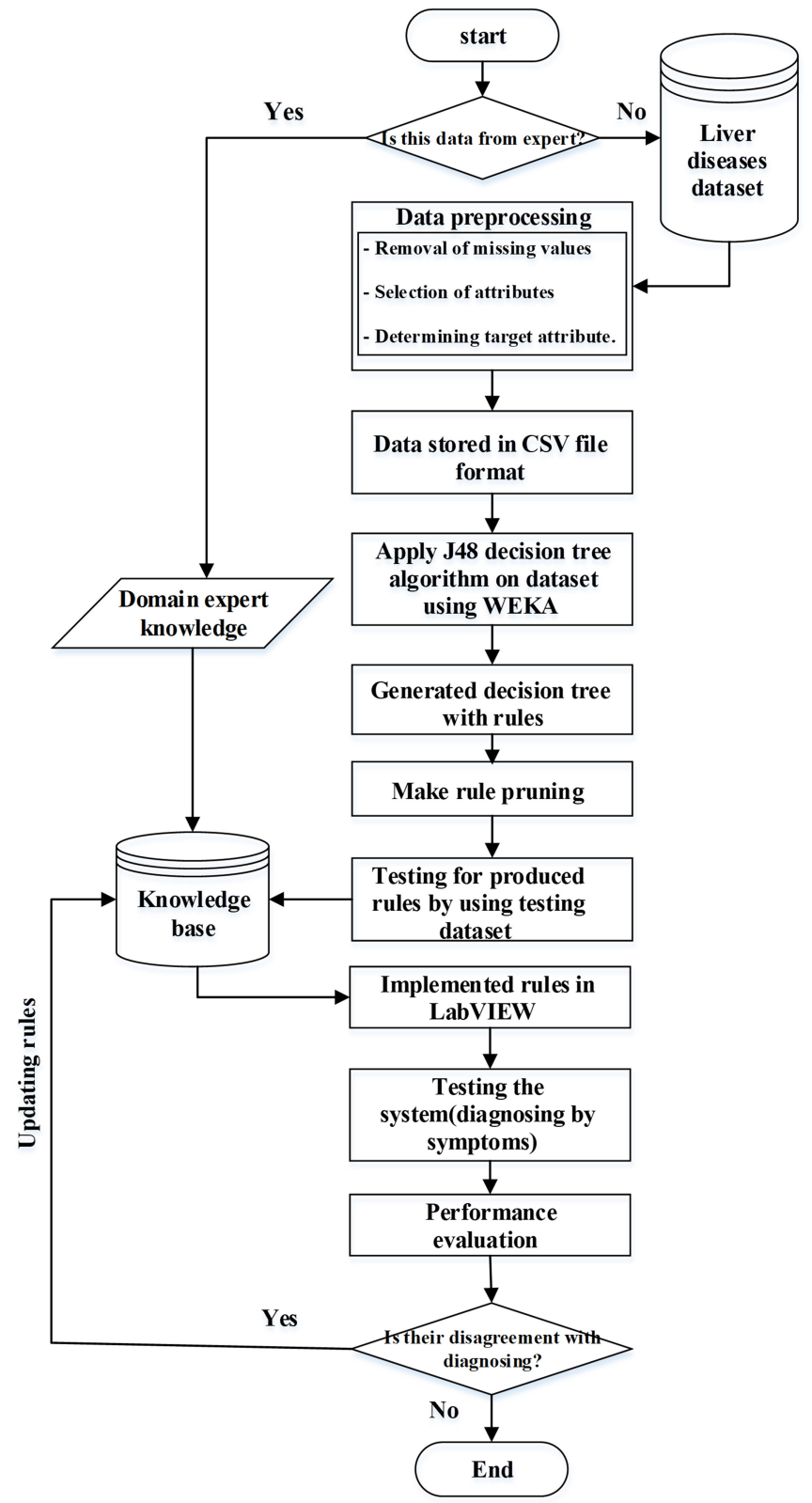

Figure 2. Flowchart of symptom-based diagnosis.

data pretreatment, visual interactive analysis, and association rule mining [14]. Figure 3 shows a snapshot of the training dataset called "diagnosing liver diseases."

The classification algorithm J48 was used for building the decision tree and its rules.

The decision tree, shown in Figure 4, has six leaves, and the overall tree size is 11 .

- Six rules were implemented, based on the pruned decision tree, as follows:

(1) IF yellow_tint_to_skin_or_eyes=Yes THEN Disease="sick_
Table 1. Symptoms of the proposed liver diseases.

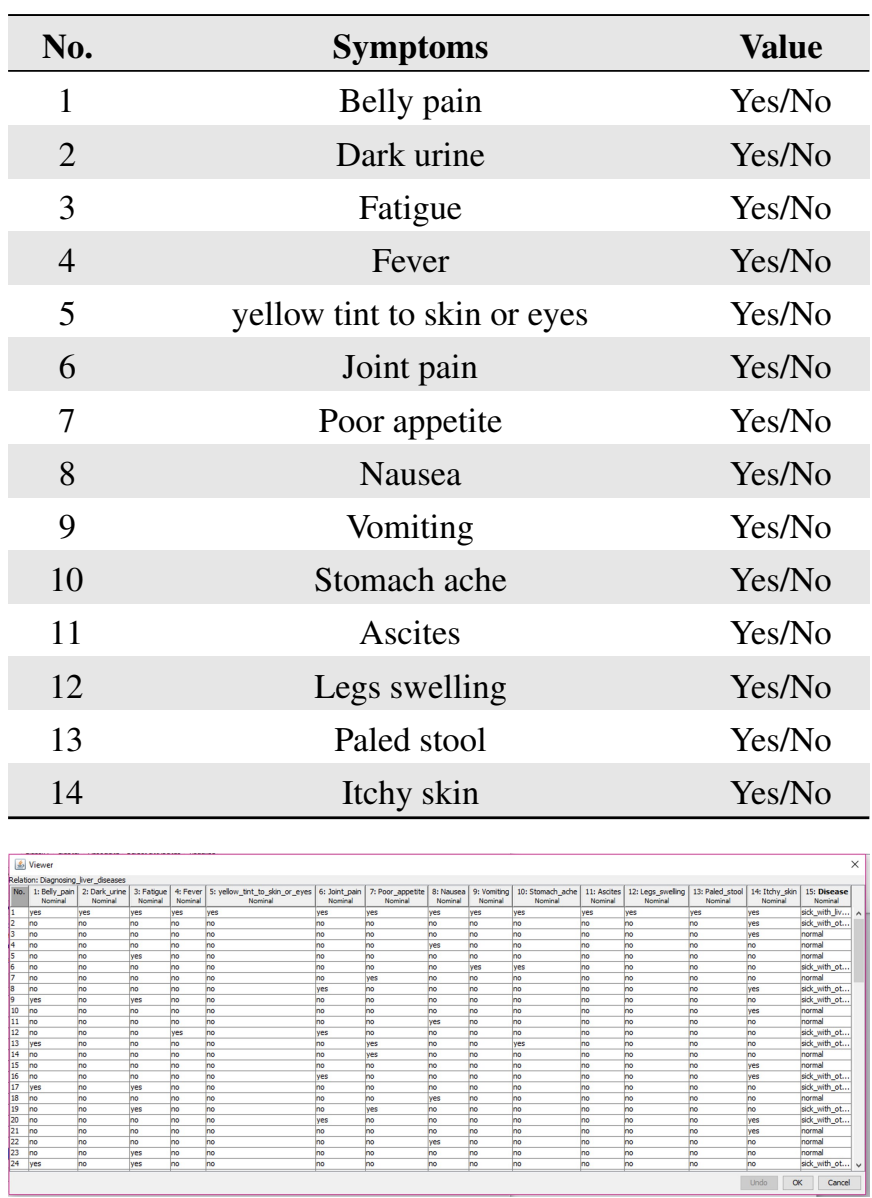

Figure 3. Snapshot of the training dataset.

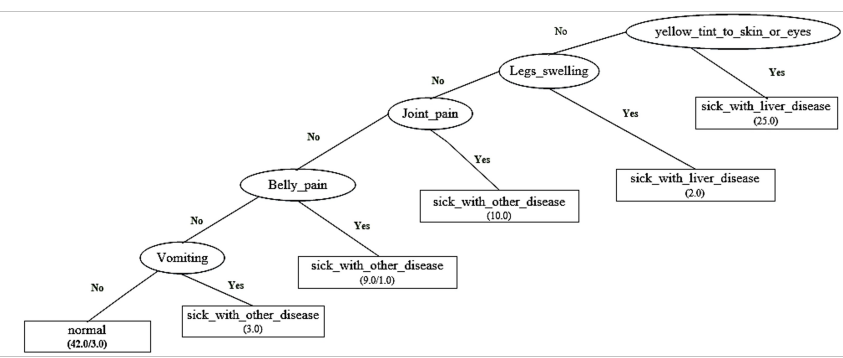

Figure 4. Decision tree for extracted rules using the J48 algorithm.

with_liver_disease"

(2) IF yellow_tint_to_skin_or_eyes $=$ No $\wedge$ Legs_swelling $=$ Yes THEN Disease="sick_with_liver_disease"

(3) IF yellow_tint_to_skin_or_eyes $=$ No $\wedge$ Legs_swelling $=$ No $\wedge$ Joint_pain $=$ Yes THEN Disease="sick_with_other_disease"

(4) IF yellow_tint_to_skin_or_eyes $=$ No $\wedge$ Legs_swelling $=$ No $\wedge$ Joint_pain $=$ No $\wedge$ Belly_pain $=$ Yes THEN Disease $=$ "sick 
with_other_disease"

(5) IF yellow_tint_to_skin_or_eyes $=$ No $\wedge$ Legs_swelling $=$ No $\wedge$ Joint_pain $=$ No $\wedge$ Belly_pain $=$ No $\wedge$ Vomiting $=$ Yes THEN Disease="sick_with_other_disease"

(6) IF yellow_tint_to_skin_or_eyes $=$ No $\wedge$ Legs_swelling $=$ No $\wedge$ Joint_pain $=$ No $\wedge$ Belly_pain $=$ No $\wedge$ Vomiting $=$ No THEN Disease $=$ "normal"

These rules were strengthened by drawing on the expertise of a number of domain experts, and adding four more rules, bringing the number of rules to 10 .

(7) IF yellow_tint_to_skin_or_eyes $=$ Yes $\wedge$ Dark_urine $=$ Yes $\wedge$ Ascites $=$ Yes $\wedge$ Paled_stool $=$ Yes $\wedge$ Itchy_skin $=$ Yes $\wedge$ Legs swelling =Yes THEN Disease ="sick_with_liver_disease"

(8) IF Fever $=$ Yes $\wedge$ Joint_pain $=$ Yes $\wedge$ Fatigue $=$ Yes THEN Disease ="sick_with_other_disease"

(9) IF Fatigue $=$ Yes $\wedge$ Itchy_skin $=$ Yes $\wedge$ THEN Disease $=$ “ Normal'

(10) IF yellow_tint_to_skin_or_eyes $=$ Yes $\wedge$ Belly_pain $=$ Yes $\wedge$ Ascites $=$ Yes $\wedge$ Stomach $\_$ache $=$Yes $\wedge$ Poor_appetite $=$ Yes $\wedge$ Nausea $=$ Yes THEN Disease="sick_with_liver_disease"

The laboratory virtual instrument engineering workbench (LabVIEW17) is a platform for system design and a development environment that utilizes a visual programming language[15]; this environment was utilized in the present work. Figure 5 shows the block diagram for symptom-based diagnosis.

A graphical user interface (GUI) was developed, for facilitating the presentation of an appropriate diagnosis. Figure 6 shows the front panel of the GUI for symptom-based diagnosis.

\subsection{Liver Function-Based Diagnosis}

Basic information about liver function tests was obtained using a database that contained records pertaining to liver disease patients previously diagnosed by more than one domain expert at Mansoura University Hospital, Department of Internal Diseases. Figure 7 shows the flowchart of the liver function test-based diagnosis process.

The rationale of the fuzzy logic approach was laid out by Professor Lotfi Ahmad Zadeh, at the University of Berkeley, California, in his seminal paper "Fuzzy Sets". Fuzzy logic is a multi-valued logic that originates from the theory of fuzzy

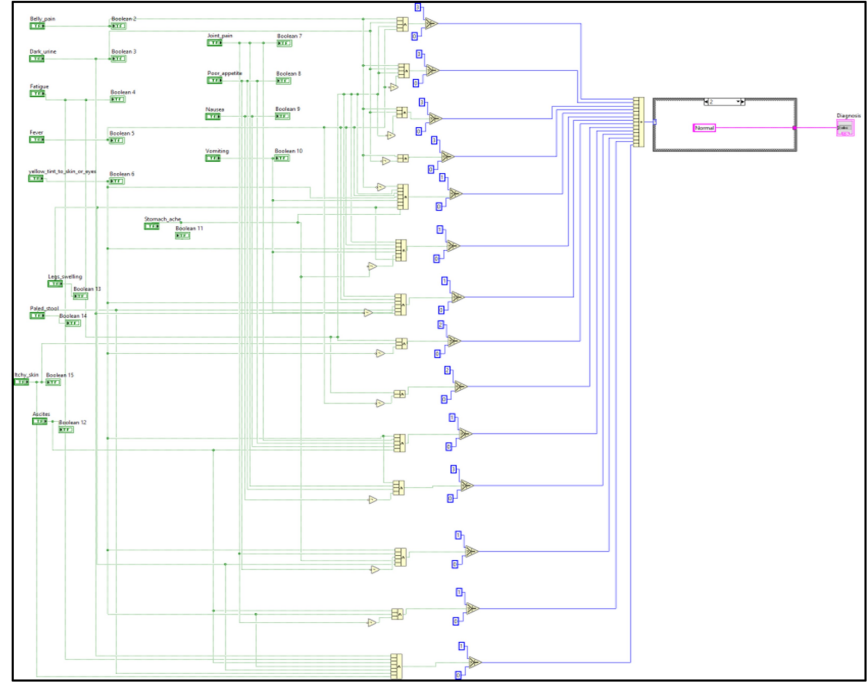

Figure 5. Block diagram of instances used in the symptom-based diagnosis.

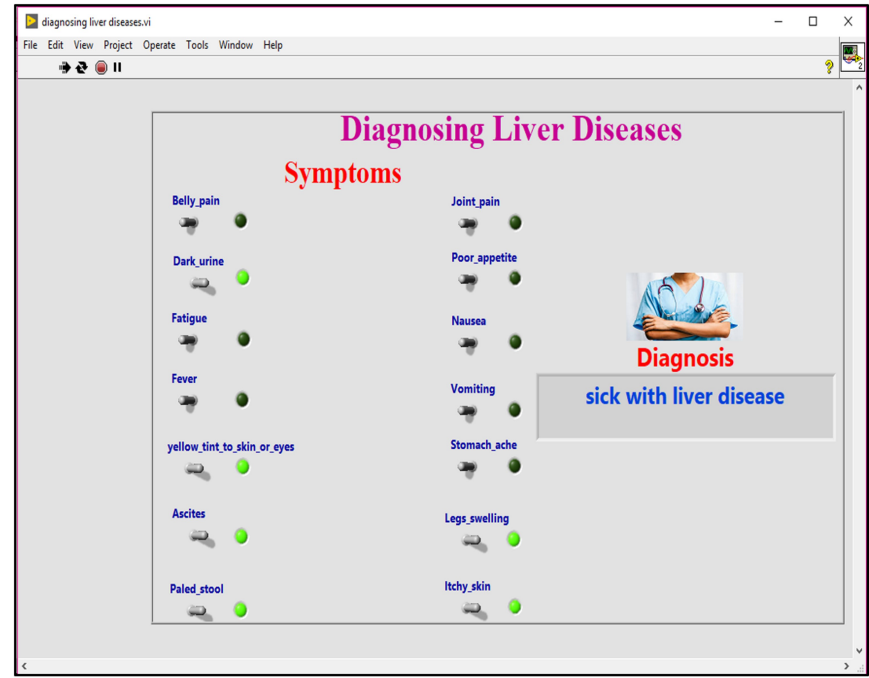

Figure 6. The front panel of the GUI for symptom-based diagnosis.

groups, for approximate reasoning. It naturally lends itself to treating linguistic information and subjective features in realworld problems[1]. This approach summarizes information with approximate behavior and describes the possible solutions in the form of fuzzy sets rather than fixed numbers[3].

A typical process of disease diagnosis has many levels of uncertainty and imprecision[16]; an example is shown in Figure 7.

In our current fuzzy DSS design, there are six input (crisp) variables and one output (crisp) variable.

Fuzzification refers to the process of transforming a crisp set into a fuzzy group. The process transforms the values of crisp 


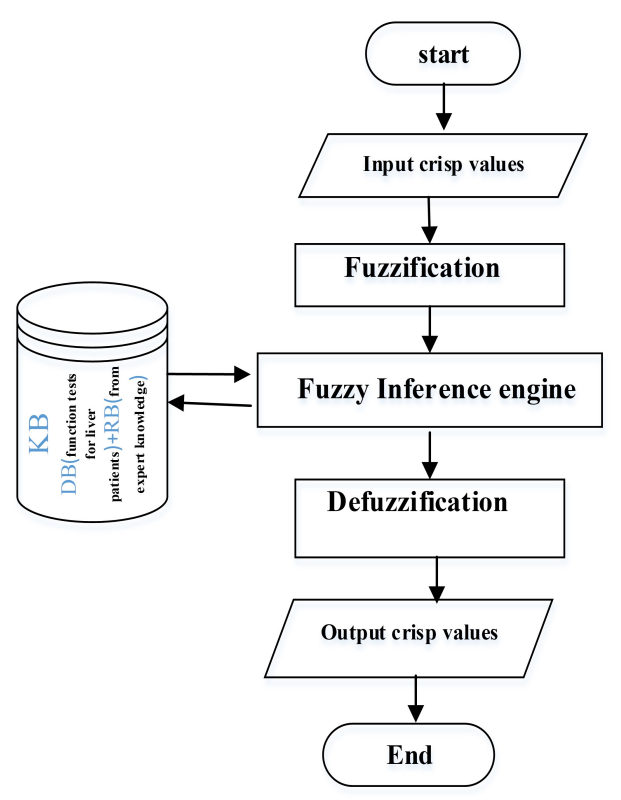

Figure 7. Fuzzy logic flowchart for the liver function test-based diagnosis process.

Table 2. Fuzzy set ranges of inputs

\begin{tabular}{|c|c|c|c|c|}
\hline No. & Inputs & Description & Fuzzy sets & Range \\
\hline \multirow{3}{*}{1} & \multirow{3}{*}{$\begin{array}{c}\text { Alanine } \\
\text { Aminotransferase }\end{array}$} & \multirow{3}{*}{$\mathrm{A} \_\mathrm{L}_{-} \mathrm{T}$} & nothing & $<7$ \\
\hline & & & normal & $7-55$ \\
\hline & & & high & $>55$ \\
\hline \multirow{3}{*}{2} & \multirow{3}{*}{$\begin{array}{c}\text { Aspartate } \\
\text { Aminotransferase }\end{array}$} & \multirow{3}{*}{ A_S_T } & nothing & $<8$ \\
\hline & & & normal & $8-48$ \\
\hline & & & high & $>48$ \\
\hline \multirow{3}{*}{3} & \multirow{3}{*}{$\begin{array}{c}\text { Alkaline } \\
\text { Phosphatase }\end{array}$} & \multirow{3}{*}{$A \perp \_P$} & nothing & $<40$ \\
\hline & & & normal & $40-129$ \\
\hline & & & high & $<129$ \\
\hline \multirow{3}{*}{4} & \multirow{3}{*}{ Bilirubin } & & nothing & $<0.1$ \\
\hline & & & normal & $0.1-1.2$ \\
\hline & & & High & $>1.2$ \\
\hline \multirow{3}{*}{5} & \multirow{3}{*}{ Prothrombin time } & \multirow{3}{*}{ P_T } & nothing & $<9.4$ \\
\hline & & & normal & $9.4-12.5$ \\
\hline & & & high & $>12.5$ \\
\hline \multirow{3}{*}{6} & \multirow{3}{*}{ Albumin } & \multirow{3}{*}{ - } & nothing & $>5$ \\
\hline & & & normal & $3.5-5$ \\
\hline & & & Low(damage. & $<3.5$ \\
\hline
\end{tabular}

input into linguistic concepts, using convenient membership functions. Table 2 lists the fuzzy set ranges of inputs, and figure 8 shows the fuzzy logic designer for the proposed system.

The inference engine and the rule base have to be specified as

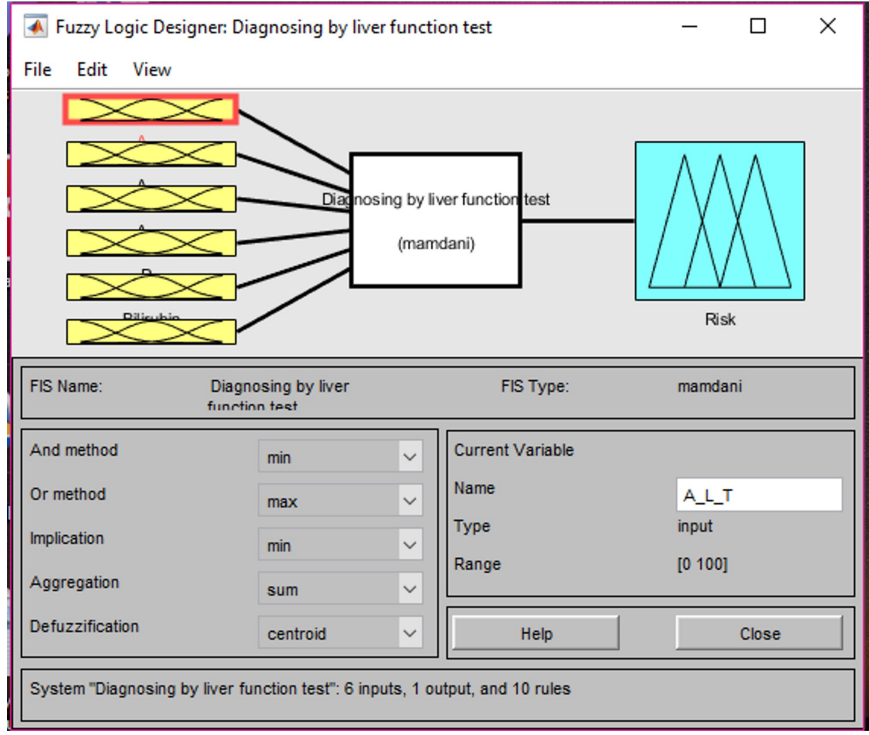

Figure 8. Fuzzy logic designer.

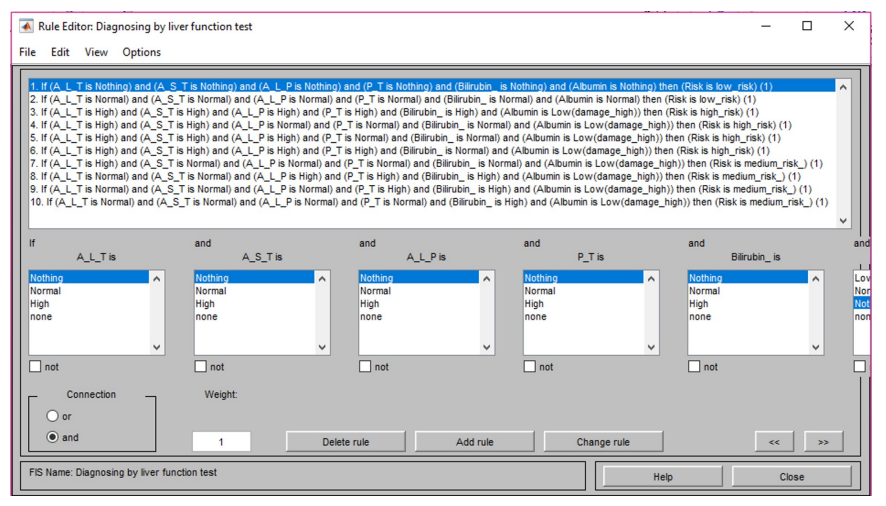

Figure 9. The rule editor.

well. After input fuzzification, groups of corresponding input fuzzified values are passed through the inference engine that handles inputs using the rules defined by the rule base. The rule base was defined by domain experts at Mansoura University Hospital, Department of Internal Diseases. In addition, an analytical laboratory was named by Dr. Farha El-Shennawi, Mansoura, Egypt. The rule base comprises 10 rules that define the percentage risk of liver damage (low risk, medium risk, and high ?risk), based on the input variables. Figure 9 shows the? rules in the rule editor.

Next, the defuzzification process takes place. In the output of the fuzzy inference process, there can be a fuzzy set that is gained by the structure of the fuzzy sets output by every rule. For practical applications, it should be linked to the crisp scope by the defuzzifier, using continent membership functions. The system uses the Mamdani model for inference, including the 


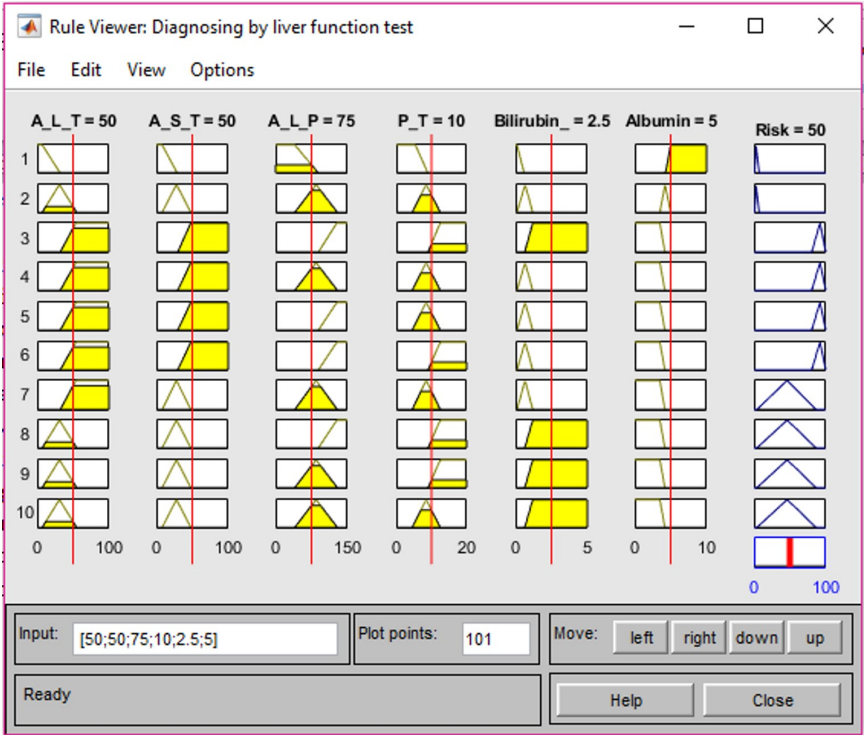

Figure 10. The resulting rules of the proposed system.

determination of the consequence as an output fuzzy set. The output fuzzy set and the aggregation method between rules are minimum for connecting the output fuzzy set; therefore, the method of fuzzification is min-max and the method of defuzzification is centroid. Figure 10 shows the resulting rules of the proposed system.

Finally, the output variable identifies the risk status of patients with liver cancer diseases. The value of the output variable is in the 0-100 range, capturing low risk, medium risk, and high-risk regimes.

\subsection{Image Processing-based Diagnosis}

This approach seeks to enhance images acquired using cameras or sensors on satellites, space probes, or medical imaging devices[17]. Image processing is another important component ?of the proposed system, and can be divided into two main phases: 1) the training phase and 2) the testing phase, as shown in Figure 11.

In the training phase, image acquisition, image pre-processing, and image feature extraction are performed.

In the image acquisition step, images associated with liver diseases are classified as computed tomography (CT), magnetic resonance imaging (MRI), or ultrasound, depending on the acquisition device. The images for the training pocess were acquired at the Department of Radiology at Mansoura ?University, radiology centers Eleman and Elmogi in Mansoura, ?and were also downloaded from publicly available repositories on the Internet.

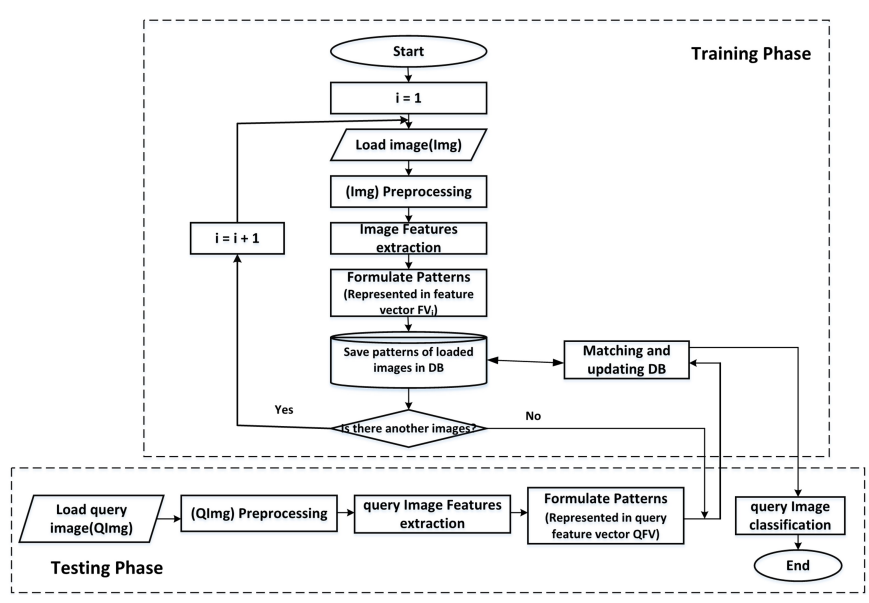

Figure 11. Flowchart for diagnosing liver disease images.

Fuzzy Decision Support System to Diagnose Liver Diseases

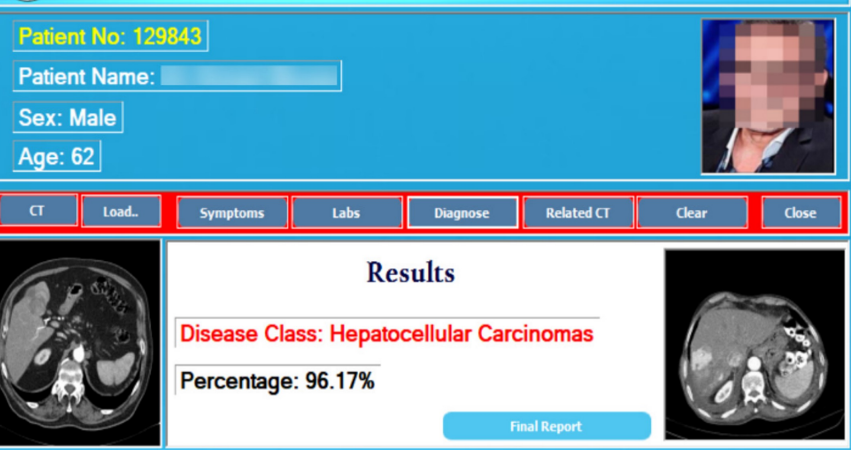

Figure 12. GUI for diagnosing liver disease images.

The acquired images were presented to ?five domain experts, for reviewing their classification and dividing them into six classes. Table 3 lists samples of some liver disease images.

During the image pre-processing step, the images are resized ?and enhanced, which helps to alleviate the effects of noise and corruption. Figure 12 shows the GUI for diagnosis using some liver disease images, including the query image and the five images most similar to the query image.

At its completion, this step yields the disease name, retrieved images related to the query image, and the percentage of similarity between a query image and images in the database.

The feature extraction technique for images is based on the gray level co-occurrence matrix (GLCM), which is a classical technique that affects image recognition, image retrieval, image segmentation, and image classification[18].

The main four extracted features using the GLCM are:

- Contrast

Contrast is a local difference computed on the GLCM. This is exemplified by a linear reliance on the gray levels of neigh- 
Table 3. Example liver disease images.

\begin{tabular}{|c|c|c|}
\hline No. & Images & Class name \\
\hline 1 & & Chronic hepatitis \\
\hline 2 & & Fatty liver \\
\hline 3 & & liver Cancer \\
\hline 4 & & Liver cirrhosis \\
\hline 5 & & Liver fibrosis \\
\hline 6 & & Normal \\
\hline
\end{tabular}

boring pixels. The contrast measure is defined by

$$
\text { Contrast }=\sum_{i, j}|i-j|^{2} P(i, j),
$$

where $i$ and $j$ are the vertical and horizontal cell coordinates, respectively, and $\mathrm{p}$ is the cell value.

- Homogeneity

Homogeneity is a very important feature. It uses the inverse of the contrast weight-to-weight values. In addition, it measures the level of uniformity of the non-zero entries in the GLCM.

$$
\text { Homogeneity }=\sum_{i, j} \frac{1}{1-(i-j)^{2}} P(i, j),
$$

Energy represents the opposite of the entropy, and is a measure of local homogeneity. Simply put, this feature quantifies the texture uniformity:

$$
\text { Energy }=\sum_{\mathrm{i}, \mathrm{j}} \mathrm{p}(\mathrm{i}, \mathrm{j})^{2}
$$

In the above, $i$ and $j$ are the row and column numbers in the GLCM.
- Correlation is defined as

$$
\text { textCorrelation }=\sum_{\mathrm{i}=0}^{\mathrm{G}=1} \sum_{\mathrm{j}=0}^{\mathrm{G}=1} \frac{\mathrm{ijP}(\mathrm{i}, \mathrm{j})-\left(\mathrm{m}_{\mathrm{i}} \mathrm{m}_{\mathrm{j}}\right)}{\sigma_{\mathrm{i}} \sigma_{\mathrm{j}}},
$$

where $\sigma_{i}$ and $\mathrm{m}_{\mathrm{i}}$ are the mean and standard deviation of $\mathrm{P}(\mathrm{i}, \mathrm{j})$ rows, $\mathrm{m}_{\mathrm{j}}$ and $\sigma_{\mathrm{j}}$ the mean and standard deviation of $\mathrm{P}(\mathrm{i}, \mathrm{j})$ ) columns, respectively[19].

The previous features can be included in the next feature vector:

$$
\mathrm{FV}_{i}=[\mathrm{E}, \mathrm{C}, \mathrm{COR}, \mathrm{H}]
$$

where $\mathrm{FV}_{i}$ is a feature vector, $i$ is the number of images, $\mathrm{E}$ is the energy, $\mathrm{C}$ is the contrast, $\mathrm{COR}$ is the correlation, and $\mathrm{H}$ is the homogeneity.

The above steps are performed until all of the chest X-ray images are considered. Then, all of the obtained patterns are saved in a database for pattern learning.

During the testing phase, a query image is received for analysis. Then, the features of this image are extracted into a query feature vector:

$$
\mathrm{QFV}=[\mathrm{E}, \mathrm{C}, \mathrm{COR}, \mathrm{H}]
$$

The WED technique is used for the matching process, which comprises the following steps:

- Finding the WED among the QFV and all vectors $\mathrm{FV}_{i}$.

- The formula of the WED measure is demonstrated as follows[19]:

$$
\mathrm{d}\left(v, v^{k}\right)=\sqrt{\sum_{i=1}^{n} p_{i\left(v_{i}-v_{i}^{k}\right)^{2}}}
$$

where

$v_{i}$ balances variations in the dynamic range, $p_{i}$ is the weight added to the component,

$k$ is the matched image index,

$$
p_{i}=\frac{N}{\int_{k-1}^{N}\left(v_{i}^{k}-\overline{v_{i}}\right)^{2}},
$$

$N$ is the number of images in the databases.

$$
\bar{v}_{i}=\frac{\sum_{K=1}^{N} v_{i}^{k}}{N} .
$$


The WED values are sorted.

- The output is the image that yields the smallest WED value.

\section{Application and Experimental Results}

The proposed system was implemented in Visual Studio.net 2017, which was used for designing the main GUI; in Matlab 17 , which was used for diagnosis using image processing and liver function tests. LabVIEW17 was used for symptom-based diagnostics. Figure 13 shows the main GUI of the proposed system.

To test and evaluate the performance of the proposed system, 30 patients with liver disease were presented to three domain experts for diagnosis. They were diagnosed using the proposed system, and the system's diagnoses were compared to the experts' diagnoses. Table 4 lists the basic evaluation metrics for diagnosing some liver diseases. Table 5 shows the confusion

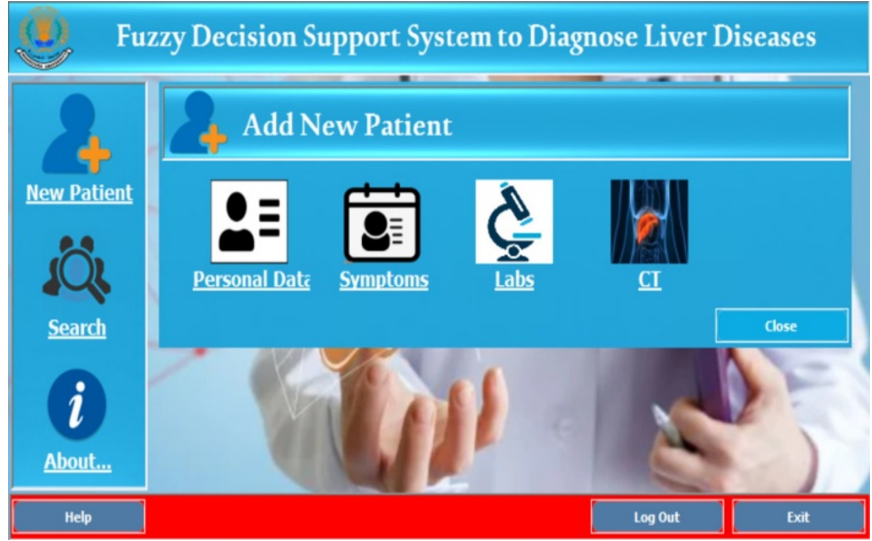

Figure 13. GUI of the proposed system.

matrix.

$$
\begin{aligned}
& \text { Accuracy }=\frac{\text { sum of correct classifications }}{\text { Total number of classifications }} \times 100, \\
& \text { Accuracy }=0.95 \% .
\end{aligned}
$$

Accuracy is the overall accuracy for image-based diagnosis.

Table 4. Basic evaluation metrics for diagnosing some liver diseases

\begin{tabular}{ccccccccc}
\hline $\begin{array}{c}\text { Disease } \\
\text { name }\end{array}$ & TP & TN & FP & FN & Precision & Sensitivity & Specificity & F-measure \\
\hline $\begin{array}{c}\text { Hepatitis } \\
\text { Liver } \\
\text { cirrhosis }\end{array}$ & 9 & 50 & 0 & 1 & 1.00 & 1.00 & 1.00 & 1.00 \\
$\begin{array}{c}\text { Liver } \\
\text { fibrosis }\end{array}$ & 10 & 50 & 0 & 0 & 1.00 & 1.00 & 1.00 & 1.00 \\
\hline $\begin{array}{c}\text { Fatty } \\
\text { liver }\end{array}$ & 10 & 49 & 1 & 0 & 0.91 & 0.91 & 0.91 & 0.91 \\
\hline $\begin{array}{c}\text { Liver } \\
\text { cancer }\end{array}$ & 10 & 39 & 1 & 0 & 0.91 & 0.91 & 0.91 & 0.91 \\
\hline Normal & 10 & 50 & 1 & 0 & 0.91 & 0.91 & 0.91 & 0.91 \\
\hline
\end{tabular}

Table 5. Confusion matrix for diagnosing some liver diseases

\begin{tabular}{ccccccc}
\hline & Hepatitis & Liver cirrhosis & Liver fibrosis & Fatty liver & Liver cancer & Normal \\
\hline Hepatitis & 9 & 0 & 0 & 1 & 0 & 0 \\
Liver cirrhosis & 0 & 8 & 0 & 0 & 1 & 1 \\
\hline Liver fibrosis & 0 & 0 & 10 & 0 & 0 & 0 \\
Fatty liver & 0 & 0 & 0 & 10 & 0 & 0 \\
Liver cancer & 0 & 0 & 0 & 0 & 10 & 0 \\
Normal & 0 & 0 & 0 & 0 & 0 & 10 \\
\hline
\end{tabular}


Error Rate $=1-$ Accuracy,

Error Rate $=1-0.95=0.05 \%$.

Error Rate is the overall error rate for image-based diagnosis.

\section{Conclusions and Future Work}

The proposed system can be used for confirming doctors' decisions. According to the experimental results, the system achieves high ?accuracy, which increases the efficiency of the system and its validity for use. Previous studies mostly employed symptom-based diagnostics, while others focused on images in the diagnostic process. These observations make the currently proposed system more distinctive and unique, owing to its ability to use both the disease symptoms and images for diagnosis, and owing to its diversity in the use of methodologies, ?algorithms, and tools for design. In addition, the proposed system has a simple and easy GUI.

The system's performance can be improved in future work by extending and designing a large knowledge base for other liver diseases, extending the currently ?proposed system to other fields, categorizing, integrating image processing, sound processing, and knowledge base in building a diagnostic fuzzy decision support system.

\section{Acknowledgements}

The authors are grateful for the support they received from the Department of Internal Diseases and the Department of Radiology at Mansoura University Hospital. The authors also would like to thank Dr. Farha El-Shennawi's analytical laboratory in Mansoura, Egypt.

\section{References}

[1] S. Subbulakshmi, G. Marimuthu, and N. Neelavathy, "A fuzzy logic decision support system for the diagnosis of heart disease," IOSR Journal of Engineering, vol. 8, no. 8, pp. 70-77, 2018.

[2] G. Improta, V. Mazzella, D. Vecchione, S. Santini, and M. Triassi, "Fuzzy logic-based clinical decision support system for the evaluation of renal function in posttransplant patients," Journal of Evaluation in Clinical Practice, vol. 26, no. 4, pp. 1224-1234, 2020. https: //doi.org/10.1111/jep.13302
[3] M. R. Farokhzad and L. Ebrahimi, "A novel adaptive neuro fuzzy inference system for the diagnosis of liver disease," International Journal of Academic Research in Computer Engineering, vol. 1, no. 1, pp. 61-66, 2016.

[4] M. Rajabi, H. Sadeghizadeh, Z. Mola-Amini, and N. Ahmadyrad, "Hybrid adaptive neuro-fuzzy inference system for diagnosing the liver disorders," 2019, Available https://arxiv.org/abs/1910.12952

[5] G. Ahmad, M. A. Khan, S. Abbas, A. Athar, B. S. Khan, and M. S. Aslam, "Automated diagnosis of hepatitis b using multilayer mamdani fuzzy inference system," Journal of Healthcare Engineering, vol. 2019, article no. 6361318, 2019. https://doi.org/10.1155/2019/6361318

[6] W. Ahmad, A. Ahmad, A. Iqbal, M. Hamayun, A. Hussain, G. Rehman, S. Khan, U. U. Khan, D. Kahn, and L. Huang, "Intelligent hepatitis diagnosis using adaptive neuro-fuzzy inference system and information gain method," Soft Computing, vol. 23, no. 21, pp. 1093110938, 2019. https://doi.org/10.1007/s00500-018-3643-6

[7] M. Mirmozaffari, "Developing an expert system for diagnosing liver diseases," European Journal of Engineering Research and Science, vol. 4, no. 3, pp. 1-5, 2019. https://doi.org/10.24018/ejers.2019.4.3.1168

[8] A. Singh and B. Pandey, "An efficient diagnosis system for detection of liver disease using a novel integrated method based on principal component analysis and knearest neighbor (PCA-KNN)," International Journal of Healthcare Information Systems and Informatics, vol. 11, no. 4, article no. 3, 2016. https://doi.org/10.4018/IJHISI. 2016100103

[9] A. Hashmia and M. S. Khanb, "Diagnosis blood test for liver disease using fuzzy logic," International Journal of Sciences: Basic and Applied Research, vol. 20, no. 1, pp. 151-183, 2015.

[10] S. Satarkar, "Fuzzy expert system for the diagnosis of common liver disease," International Engineering Journal for Research \& Development, vol. 1, no. 1, pp. 2014.

[11] S. Kim, S. Jung, Y. Park, J. Lee, and J. Park, "Effective liver cancer diagnosis method based on machine learning algorithm," in Proceedings of 2014 7th International Conference on Biomedical Engineering and Informatics, 
Dalian, China, 2014, pp. 714-718. https://doi.org/10.1109/ BMEI.2014.7002866

[12] M. Neshat, A. Masoumi, M. Rajabi, and H. Jafari, "Diagnosing hepatitis disease by using fuzzy hopfield neural network," Annual Research \& Review in Biology, vol. 4, no. 17, pp. 2709-2721, 2014. https://doi.org/10.9734/ ARRB/2014/9183

[13] M. Adeli, N. Bigdeli, and K. Afshar, K. (2013, May). New hybrid hepatitis diagnosis system based on Genetic algorithm and adaptive network fuzzy inference system. In 2013 21st Iranian conference on electrical engineering (ICEE), Mashhad, Iran, 2013, pp. 1-6. https://doi.org/10. 1109/IranianCEE.2013.6599872

[14] S. Kodati and R. Vivekanandam, "Analysis of heart disease using in data mining tools Orange and Weka," Global Journal of Computer Science and Technology, vol. 18, no. 1C, pp. 17-21, 2018.

[15] M. Mahmoodi, L. A. James, and T. Johansen, "Automated advanced image processing for micromodel flow experiments; an application using LabVIEW," Journal of Petroleum Science and Engineering, vol. 167, pp. 829-843, 2018. https://doi.org/10.1016/j.petrol.2018.02.031

[16] A. Torres and J. J. Nieto, "Fuzzy logic in medicine and bioinformatics," Journal of Biomedicine and Biotechnology, vol. 2006, article no. 091908, 2006. https://doi.org/ $10.1155 / \mathrm{JBB} / 2006 / 91908$

[17] P. Ravi and A. Ashokkumar, "Analysis of various image processing techniques," International Journal of Advanced Networking \& Applications, vol. 8, no. 5, pp. 8689, 2017.

[18] X. Zhang, J. Cui, W. Wang, and C. Lin, "A study for texture feature extraction of high-resolution satellite images based on a direction measure and gray level co-occurrence matrix fusion algorithm," Sensors, vol. 17, no. 7, article no. 1474, 2017. https://doi.org/10.3390/s17071474

[19] A. E. E. Elalfi, H. Fouda, and A. Atta, "Developing an Intelligent decision support system for the diagnosis of some children's diseases," Computer Science Heart and Chest Surgery Department Mansoura University, vol. 151, no. 2 , pp. 32-38, 2016. http://dx.doi.org/10.5120/ ijca2016911688

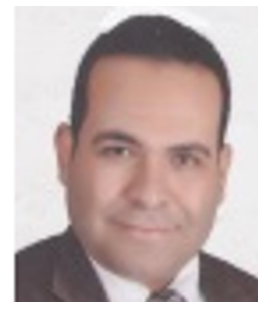

Ahmed Abd El-Badie Abd Allah Kamel is lecturer in the department of computer science, Faculty of Specific Education, Mansoura University, Egypt, and head of information systems and decision support department, Egyptian aviation and Space Center. His research interests include pattern recognition, machine learning, data mining, fuzzy logic, and image processing. E-mail: ahmed_abdelbadie@mans.edu.eg

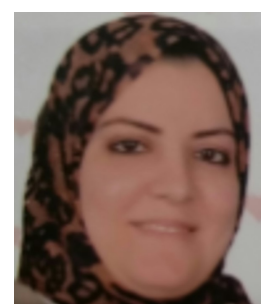

image processing.

E-mail: faten_com1@mans.edu.eg
Faten Abd El-Sattar Zahran El-Mougi is lecturer in the department of computer science, Faculty of Specific Education, Mansoura University, Egypt. Her research interests include pattern recognition, fuzzy logic, machine learning, data mining, and 